\title{
Interpretação e reconhecimento de padrões para avaliação de dor em imagens faciais de recém-nascidos
}

\author{
Pedro A. S. S. Orona ${ }^{1}$, Davi A. D. Fabbro ${ }^{1}$, Tatiany M. Heiderich ${ }^{2}$, \\ Marina C. M. Barros ${ }^{2}$, Rita C. X. Balda ${ }^{2}$, Ruth Guinsburg ${ }^{2}$, Carlos E. Thomaz ${ }^{1}$ \\ ${ }^{1}$ Departamento de Engenharia Elétrica, FEI \\ São Bernardo do Campo, São Paulo \\ ${ }^{2}$ Departamento de Medicina Neonatal, UNIFESP \\ São Paulo, São Paulo.
}

pedroaugusto.eng@gmail.com, ddfabbrodfei.edu.br, tatianyms@gmail.com
\{marinamoraesbarros, ritabalda\}@uol.com.br, ruth.guinsburg@gmail.com,
cetefei.edu.br

\begin{abstract}
Pain analysis in newborns has become a relevant study subject over the last few decades, given the inability to objectively identify the source and intensity of the pain in newborn babies. Considering this context, this work's main objective is to develop a computer framework capable of recognizing and interpreting patterns in facial expressions for an automated evaluation of pain levels on term babies. Being that, the framework developed here, get an accuracy (upper limit) of approximately $96 \%$ for the COPE base and $77 \%$ for the UNIFESP base.
\end{abstract}

Resumo. Analisar a dor em recém-nascidos se tornou um estudo de relevância nas últimas décadas dada a impossibilidade de se conseguir indicar objetivamente qual a localização e intensidade deste fenômeno doloroso. Neste contexto, o objetivo deste trabalho é desenvolver um arcabouço computacional de interpretação e reconhecimento de padrões em imagens de faces para avaliação automática de dor em bebês a termo. Sendo que, o arcabouço desenvolvido aqui, obteve uma taxa de acerto (limite superior) de aproximadamente $96 \%$ para a base COPE e $77 \%$ para a base UNIFESP.

\section{Introdução}

A dor é uma característica intrínseca a qualquer ser vivo que possua sistema nervoso central, servindo para alertar que algo está errado, uma presença de dano corporal ou ameaça de morte [Luda et al. 2007]. Clinicamente, a dor é definida como uma experiência subjetiva e o método mais confiável para avaliá-la é através do auto-relato, ou seja, por meio de expressões e indicações pontuais individuais[Taddio et al. 1997].

Para a maioria dos adultos é fácil descrever verbalmente a localização, duração e intensidade de suas experiências dolorosas, porém para o caso de recém-nascidos, é preciso uma atenção maior, dada a impossibilidade contextual de indicação e classificação de dor. Logo, para este caso, há exigência de serem desenvolvidos métodos de análise que busquem classificar a dor utilizando informações fisiológicas e comportamentais.

Tendo como maior referência e ponto de início o projeto desenvolvido por [Teruel et al. 2019], este trabalho tem como objetivo principal propor e implementar, 
através do pré-processamento e análise multivariada, um método não invasivo capaz de identificar, classificar e avaliar dor utilizando imagens faciais de bebês a termo. Foi construído aqui um arcabouço computacional baseado em detecção e segmentação de objetos, normalização espacial e classificação de imagens de faces para caracterização e quantificação multivariada [Thomaz et al. 2006] de diferenças estatisticamente significantes, tendo a etapa de normalização como um dos processos obrigatórios para a classificação de dor, a qual não foi implementada em outros trabalhos que buscam o mesmo objetivo de classificar dor utilizando imagens faciais de bebês recém-nascidos.

Estes métodos foram aplicados em duas bases de imagens distintas, uma delas disponibilizada pela UNIFESP [Heiderich et al. 2015], e outra disponível publicamente via solicitação, denominada COPE [Brahnam et al. 2006]. Ambas as bases foram construídas em ambiente controlado e são compostas por imagens faciais de bebês a termo que vivenciaram algum tipo ou nível de dor em decorrência de procedimentos clínicos tradicionais e necessários para diagnóstico e/ou tratamento de morbidades próprias do período neonatal [Heiderich et al. 2015].

Os resultados experimentais deste trabalho mostram que é possível não somente classificar estatisticamente dor e não-dor através de imagens de face, mas também evidenciar regiões faciais discriminantes para o fenômeno dor, auxiliando na construção de escalas pediátricas de dor mais gerais e assertivas.

As demais seções deste artigo podem ser assim resumidas. Na seção 2 seguinte, é descrita em detalhes a metodologia implementada, que contém como etapas principais a seleção de imagens, normalização espacial das mesmas, extração multivariada de informações discriminantes e a descrição dessas informações em mapas estatísticos. Depois, na seção 3, são apresentados os experimentos e resultados para as duas bases de imagens selecionadas, sendo uma dessas bases de domínio público. Por fim, na seção 4, conclui-se o artigo com perspectivas de trabalhos futuros a partir dos achados descritos neste trabalho.

\section{Metodologia}

Esta seção descreve em detalhes a metodologia proposta e implementada neste trabalho, composta das seguintes etapas principais: normalização espacial das imagens, extração de características e análise estatística multivariada, descritas na figura 1.

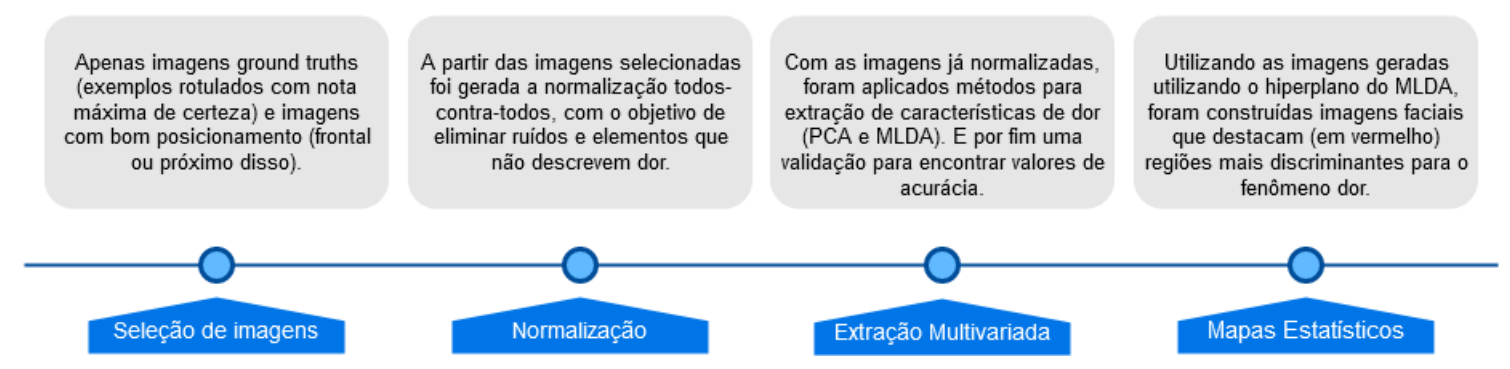

Figura 1. Etapas principais da metodologia.

Para a normalização espacial das imagens, implementou-se um framework computacional que combina posicionamento de pontos de referências (landkmarks) faciais, registro não-rígido de imagens, utilizando funções triangulares, e construção de atlas 
inéditos de faces de recém-nascidos [Gibson 2006]. Para extração de características, utilizou-se o PCA (Análise das Componentes Principais), para redução de dimensionalidade e interpretação do conjunto de dados. Após a redução de dimensionalidade, aplicou-se o MLDA (Multi-Linear Discriminant Analysis), com o intuito de encontrar as características mais discriminantes e, por fim, classificar as imagens entre "Dor" e "NãoDor".

\subsection{Seleção de imagens}

Foram escolhidas apenas os ground truths para realizar o processo de normalização de todos-contra-todos e construção dos hiperplanos, ou seja, somente as imagens rotuladas pelos especialistas com nota máxima de certeza como dor e não-dor na escala NFCS entraram nesta seleção. Ground truth é importante para classificação supervisionada das imagens, pois podemos garantir uma maior consistência do classificador construído, dado que estamos tratando de casos extremos, dor e não-dor, e retirando os casos de incerteza.

Foram também removidas da seleção final para construção do hiperplano as imagens que estavam prejudicadas de alguma maneira, seja por mal posicionamento da câmera no momento da captura, por artefatos posicionados sobre a face do recémnascidos, entre outros.

Por fim, para a base COPE, foram utilizadas 55 imagens de dor e 62 de não-dor, e para a UNIFESP foram utilizadas 21 imagens de dor e 26 de não-dor. Para o caso da base UNIFESP, foram considerados ground truths de dor apenas as imagens no instante da punção venosa, pois neste momento o recém-nascido está experienciando algum nível de dor.

\subsection{Normalização}

Para servir de entrada aos métodos de extração multivariada de informação, foram geradas novas imagens através da metodologia proposta por [Orona et al. 2019], porém aplicando a política de todos-contra-todos, em vez de um-contra-todos. Para o um-contra-todos, utiliza-se uma imagem fixa de referência e todos as normalizações utilizam esta imagem como base, já o todos-contra-todos elimina a necessidade de se utilizar uma imagem de referência.

Este método iterativo propõe que, para cada passo, uma imagem $I_{1}$ da base de dados de tamanho $N$ seja utilizada como referência, ou seja, é retirado do conjunto uma imagem, e esta é utilizada como referência para calcular a transformação das $N-1$ imagens restantes. Após isto, calcula-se a média dessas transformações e aplica-se na imagem $I_{1}$. Este processo se repete então por mais $N-1$ vezes, até que todas as imagens estejam normalizadas.

Foi escolhido este método para normalização dos dados principalmente pelo fato de que este busca eliminar o viés que uma imagem fixa de referência pode, possivelmente, criar em uma normalização um-contra-todos.

Para cada imagem $I_{i}$ escolhida como referência a normalização gera transformações $T_{i, j}$ para $j=1, \ldots, N, j \neq i$, que por fim geram uma transformação média $\overline{T_{i}}$ : 


$$
\overline{T_{i}}=\frac{1}{(N-1)} \sum_{j=1}^{N} T_{i, j}
$$

para cada imagem $i$. A imagem média $\overline{I_{i}}$ é definida como a imagem $I_{i}$ espacialmente transformada por $\overline{T_{i}}$.

\subsection{Extração Multivariada de Informações}

Para os casos em que o conjunto de dados a ser analisado inclui medições simultâneas em muitas variáveis, a metodologia empregada recebe o nome de análise multivariada [Johnson and Wichern 2006]. Este tipo de análise foi aplicada no presente trabalho com o objetivo de realizar análises conjuntas e simultâneas de duas ou mais variáveis correlacionadas entre si, provenientes de um mesmo conjunto de dados.

Logo, através da combinação de métodos lineares, PCA (Principal Component Analysis) e MLDA (Maximum uncertainty LDA-base), foram gerados hiperplanos estatístico para separar exemplos das bases de imagem entre exemplos de dor e não-dor.

\subsection{Mapas Estatísticos}

Por fim, para visualização das diferenças entre os resultados gerados para as bases COPE e UNIFESP, foram calculados mapas estatísticos denominados aqui por mapas T, selecionando as imagens de cada extremo da navegação do MLDA. Para a construção destes mapas, primeiramente, é realizada uma diferença pixel a pixel dos pares de imagens, além de uma normalização para transformar todas essas diferenças em valores positivos, definida como:

$$
m_{\text {diff }}=\sqrt{\left(I_{1}-I_{2}\right)^{2}},
$$

em que $I_{1}$ é a imagem vetorizada do grupo 1 (dor) e $I_{2}$ a do grupo 2 (não-dor).

Computado este módulo da diferença, foi calculada a divisão pixel a pixel do vetor $m_{\text {diff }}$ pelo desvio padrão global da base, tomando o cuidado de realizar este cálculo apenas nas pixels $s$, onde o desvio padrão não era zero, ou seja, $s<N$ e $\sigma_{i}(s) \neq 0$.

\section{Experimentos e Resultados}

Com o objetivo de construir um hiperplano que consiga descrever e separar imagens faciais de dor e não-dor, foram extraídas as características mais relevantes de dor, e isso se deu por meio da aplicação do PCA. A seguir, foi utilizado o MLDA para separar os dados entre as duas classes. Para o PCA foram utilizadas o número de componentes não-nulas [Fukunaga 2013], no caso da base COPE foram 116 e para a da UNIFESP foram 46.

\subsection{Geração do hiperplano}

Seguindo o processo descrito na subseção 2.2, as imagens selecionadas foram normalizadas gerando um novo conjunto de dados. Foi então obtida para a base COPE a separação 


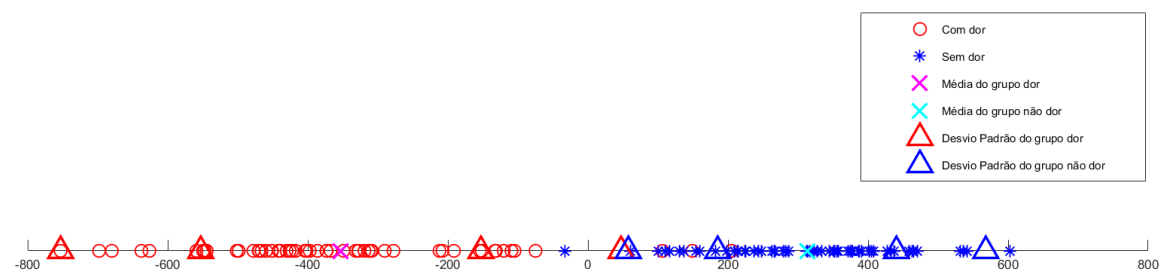

Figura 2. Projeção dos dados no eixo discriminante entre o grupo com dor (esquerda) e sem dor (direita) para a base COPE.

apresentada na Figura 2, em que os desvios padrões de cada classe foram multiplicados por $i$, onde $i \in\{-2,-1,1,2\}$. Observa-se que o algoritmo foi capaz de dividir no hiperplano discriminante as duas classes, dor e não-dor.

Para verificar as componentes geradas, foi calculada a navegação ao longo do vetor de componentes do PCA. A Figura 3 apresenta as 7 primeiras componentes de maior autovalor e suas respectivas navegações, sendo a imagem do meio a média global.

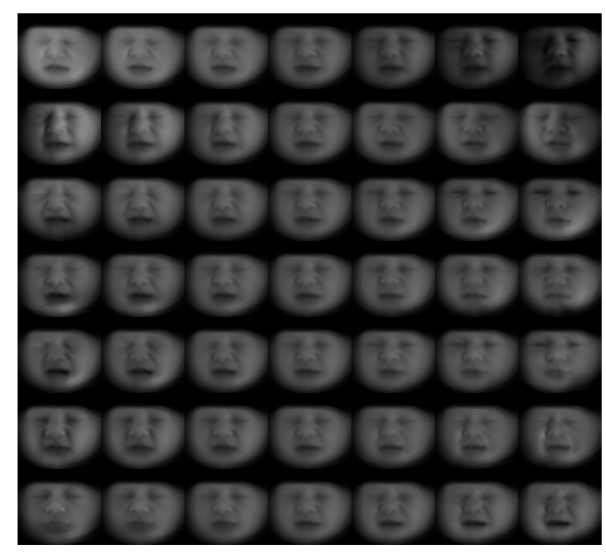

Figura 3. Navegação no hiperplano das sete primeiras componentes principais, ordenadas da maior para menor variância, para a base COPE.

É importante mencionar que o processo de normalização utilizando a metodologia de todos-contra-todos promove uma transformação menos agressiva dos dados, gerando regiões borradas principalmente no contorno da face.

Nesta imagem é possível encontrar alguns padrões de dor, como por exemplo, bocas abertas, olhos fechados e franzidos, entre outros. Também pode-se visualizar que a primeira componente provavelmente descreve artefatos globais das imagems, como iluminação e contraste, logo, pode-se realizar uma nova separação removendo-a, dado que esta componente provavelmente não descreve características relevantes para o fenômeno da dor.

Foi realizada também a navegação no hiperplano do MLDA, com o intuito de gerar imagens artificiais que representem uma atenuação ou mitigação da dor, gerando a Figura 4. Nesta, a imagem central se refere à média global e as restantes são geradas através da navegação do hiperplano, definindo os saltos desta.

Interessante notar que quanto mais caminha-se para esquerda, mais as características de dor começam a surgir, como por exemplo, testa franzida, olhos espremidos, 


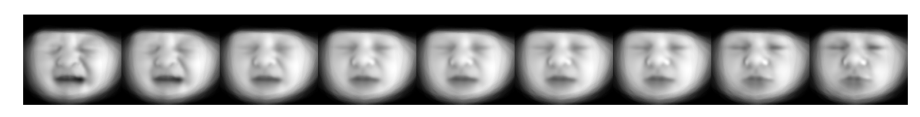

Figura 4. Navegação no hiperplano discriminante para a base COPE.

aprofundamento do suco nasolabial e boca horizontalmente aberta. Em contrapartida, quando parte-se da imagem média global e caminha-se para a direita, características de não dor se tornam mais presentes, como fechamento da boca, abertura dos olhos, relaxamento da testa e etc.

Com o intuito de buscar uma separação ainda melhor dos dados, foi removida a primeira componente e gerado um novo hiperplano de separação.

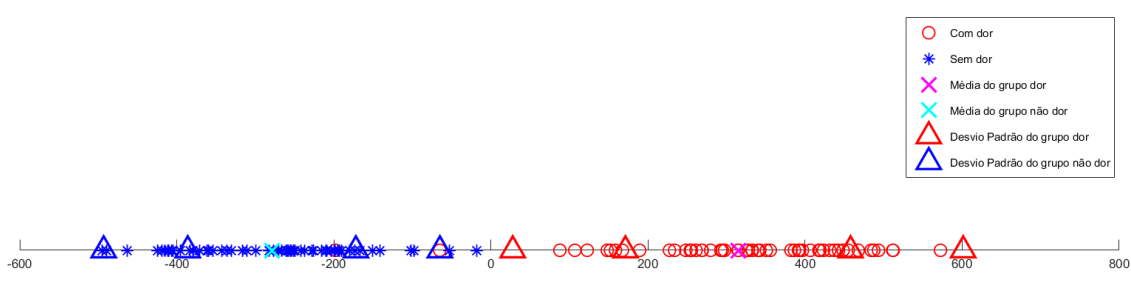

Figura 5. Projeção dos dados no eixo discriminante entre o grupo com dor e sem dor removendo a primeira componente principal para a base COPE.

Como visto na Figura 5, ocorrem menos sobreposições de imagens de classes distintas, sendo que, neste caso, indivíduos com dor foram posicionados no lado positivo do hiperplano, representado pela classe não-dor. Outro resultado interessante é que neste novo hiperplano, há uma distância maior entre os extremos das duas classes, sendo outro ponto positivo para esta separação, pois mostra que as classes estão melhor divididas.

Foi gerada também para este caso a navegação do hiperplano do MLDA, apresentada na Figura 6.

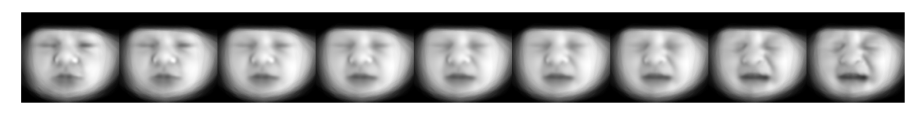

Figura 6. Navegação no hiperplano discriminante removendo a primeira componente principal original do PCA para a base COPE.

Utilizando o mesmo procedimento descrito anteriormente, foi construído o hiperplano para separação das imagens da base UNIFESP entre dor e não-dor, apresentado na Figura 7.

Nota-se que, para esta base, a separação gerou um número maior de sobreposições entre os exemplos de cada classe, diferente das separações da base COPE, esta apresenta uma sobreposição considerável, existindo uma região de dúvida entre dor e não-dor. Algumas suposições do porquê deste resultado seriam: o MLDA encontrou dificuldade em espalhar os indivíduos entre as regiões de separação linear, pois as imagens rotuladas como dor e não-dor podem, talvez, estar compartilhando características semelhantes, dificultando assim o posicionamento dos indivíduos no hiperplano; a quantidade de imagens não foi suficiente para o MLDA gerar um hiperplano assertivo. Note que para a base COPE o número de imagens foi maior que o dobro comparado com a UNIFESP. 


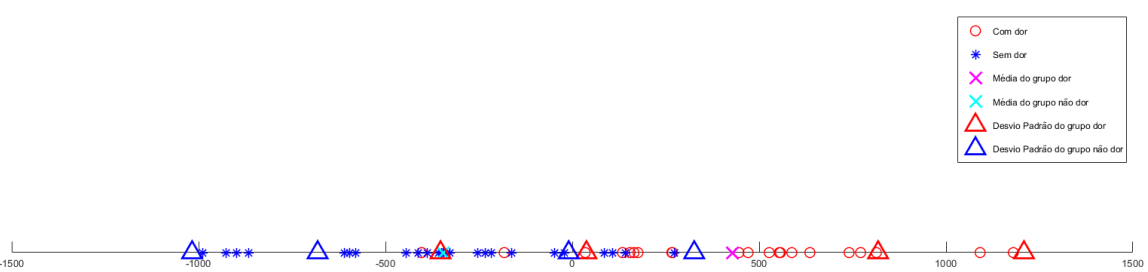

Figura 7. Projeção dos dados no eixo discriminante entre o grupo com dor (esquerda) e sem dor (direita) para a base UNIFESP.

Foram geradas também as navegações do PCA e do MLDA utilizando os vetores construídos no passo anterior, apresentadas nas Figuras 8 e 9, respectivamente.

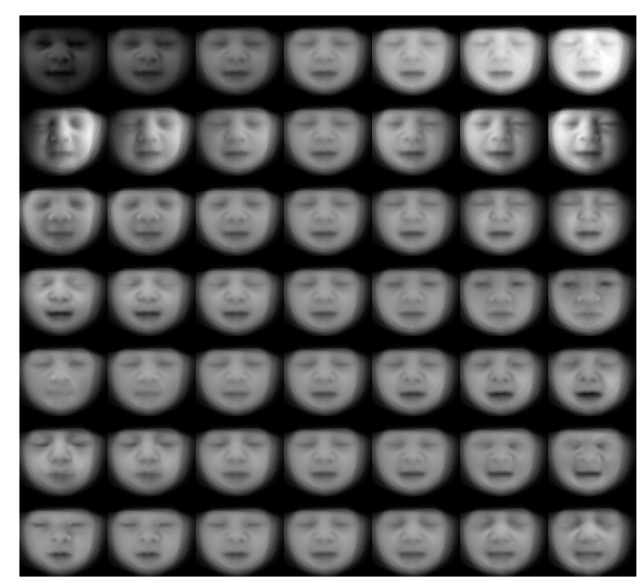

Figura 8. Navegação no hiperplano das sete primeiras componentes principais, ordenadas da maior para menor variância, para a base UNIFESP.

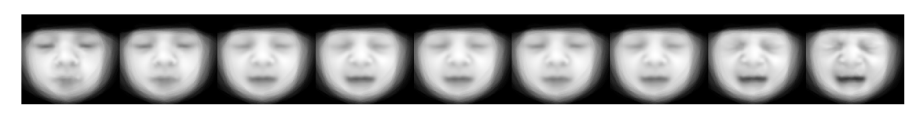

Figura 9. Navegação no hiperplano discriminante para a base UNIFESP.

Verifica-se também na análise das componentes principais que as duas primeiras componentes de maior autovalor não estão discriminando fenômenos relacionados à dor, e sim aos artefatos globais da imagem. A primeira parece descrever as variações de iluminação mais globais e a segunda variações de iluminação locais. Note que na segunda componente na imagem gerada no extremo esquerdo há uma iluminação no lado direito da face e na imagem gerada no extremo direito há uma iluminação no lado esquerdo da face, como se a componente tentasse descrever um objeto luminoso transladando ao redor da face do indivíduo.

Logo, foi aplicado novamente o PCA junto ao MLDA porém removendo essas duas componentes, além da navegação no hiperplano do MLDA, e os resultados são ilustrados nas Figuras 10 e 11.

Desta vez foi obtida uma melhor separação dos dados, ocorrendo poucos casos de sobreposição de indivíduos de classes distintas. 


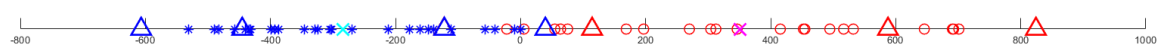

Figura 10. Projeção dos dados no eixo discriminante entre o grupo com dor e sem dor removendo a primeira componente principal para a base UNIFESP.

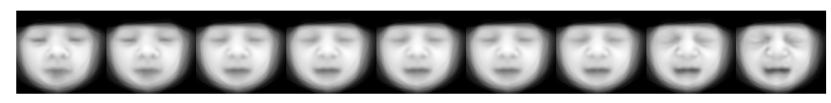

Figura 11. Navegação no hiperplano discriminante removendo a primeira componente principal original do PCA para a base COPE.

\subsection{Validação}

Com o objetivo de avaliar os hiperplanos construídos, foram utilizados 2 métodos de validação cruzada, o $k$-fold, sendo seu resultado utilizado como limite inferior para a classificação, e o leave-one-out, utilizado como limite superior.

Seguindo o procedimento padrão de classificação, ambas as bases foram separadas em conjuntos de treinamento e teste. Todos os métodos de separação foram construídos utilizando a plataforma Matlab e a função crossvalin para separar aleatoriamente as imagens entre esses conjuntos. O k-fold foi implementado com $k=5$, ou seja, foram realizadas 5 iterações sendo que $20 \%$ dos dados foram separados para teste e os $80 \%$ restantes para treinamento. Foi utilizada a distância euclidiana simples em relação ao indivíduo e às médias de cada grupo (dor e não-dor). Ao final da classificação, para cada conjunto de treinamento, foi realizado a média de todas as taxas de acerto de cada iteração. Considerando que os resultados de acurácia se referem à performance geral do modelo, ou seja, dentre todas as classificações (dor e não-dor), quantas ele classificou corretamente.

Primeiramente para a base COPE, utilizando $k$-fold e o hiperplano apresentado na Figura 4 com todas as componentes do PCA, foi obtida uma acurácia média de 83,64\%.

Já para o leave-one-out foi alcançada a acurácia média de 95,73\%, observando que houve um aumento considerável de acerto, porém devemos considerar que este é o valor máximo de classificação que o arcabouço construído pode alcançar, utilizando o hiperplano em questão.

Finalmente para o leave-one-out foi alcançada a acurácia média de 95,73\%, valor exatamente igual ao obtido utilizando o primeiro hiperplano. Portanto, para a base COPE, foi obtida uma acurácia relativamente alta, comprovando que o hiperplano construído, principalmente o segundo, pode ser utilizado para classificar imagens faciais de bebês recém-nascidos entre dor e não-dor.

Em seguida, utilizando a base UNIFESP para o processo de classificação, foi aplicado o $k$-fold com $k=5$ e o hiperplano apresentado na Figura 9, foi obtida a acurácia média de $64,17 \%$, bem menor em comparação à acurácia média obtida utilizando a base COPE, dado que a separação realizada pelo MLDA não foi tão assertiva.

Já aplicando o leave-one-out foi alcançada a acurácia média de 65,96\%, ou seja, 
para a base UNIFESP, utilizando o primeiro hiperplano gerado, chegou-se à um limite inferior de $64,17 \%$ e um superior de $65,96 \%$.

Neste caso, foi obtida uma acurácia média de 76,6\% para o leave-one-out. Esta foi a maior taxa alcançada entre os métodos, embora este seja um valor relativamente baixo. Supõe-se novamente as mesmas questões discutidas nos resultados obtidos na construção do primeiro hiperplano da base UNIFESP.

As Tabelas seguintes resumem todos os resultados obtidos para as bases COPE e UNIFESP, respectivamente, com as informações de acurácia, sensitividade, especificidade, precision e recall, sendo que para o k-fold foi calculada a média e o desvio padrão dessas métricas:

\begin{tabular}{|c|c|c|}
\hline \multicolumn{3}{|c|}{ COPE - K-Fold } \\
\hline & PCA + MLDA & PCA (1 componente a menos) + MLDA \\
\hline Acurácia & $0,8364 \pm 0,0689$ & $0,8727 \pm 0,0874$ \\
\hline Precision & $0,9457 \pm 0,0512$ & $0,9652 \pm 0,0478$ \\
\hline Recall & $0,7758 \pm 0,1073$ & $0,8292 \pm 0,1065$ \\
\hline \multicolumn{3}{|c|}{ COPE - Leave-One-Out } \\
\hline & PCA + MLDA & PCA (1 componente a menos) + MLDA \\
\hline Acurácia & 0,9573 & 0,9573 \\
\hline Precision & 0,9385 & 0,9385 \\
\hline Recall & 0,9839 & 0,9839 \\
\hline
\end{tabular}

Tabela 1. Resultados das classificações para a base COPE.

\begin{tabular}{c|c|c}
\hline \multicolumn{3}{c}{ UNIFESP - K-Fold } \\
\hline & PCA + MLDA & PCA (2 componentes a menos) + MLDA \\
\hline Acurácia & $0,6417 \pm 0,1782$ & $0,6889 \pm 0,0712$ \\
Precision & $0,7533 \pm 0,1850$ & $0,7857 \pm 0,1577$ \\
Recall & $0,6267 \pm 0,2477$ & $0,7414 \pm 0,1991$ \\
\hline \multicolumn{2}{c}{ UNIFESP - Leave-One-Out } \\
\hline & PCA + MLDA & PCA (2 componentes a menos) + MLDA \\
\hline Acurácia & 0.6596 & 0.7660 \\
Precision & 0.6786 & 0.7586 \\
Recall & 0.7308 & 0.8462 \\
\hline
\end{tabular}

Tabela 2. Resultados das classificações para a base UNIFESP.

Para a base COPE, temos resultados consideráveis comparados com os anteriores que utilizaram a mesma base, sendo que devemos levar em consideração que o limite inferior alcança acurácias bem próximas aos resultados de outros trabalhos [Brahnam et al. 2006, Mansor and Rejab 2013], e o limite superior de aproximadamente $96 \%$ alcança porcentagens de acurácia maiores que os apresentados em trabalhos anteriores, com a ressalva de que foi realizada uma separação de imagens anterior à normalização e classificação.

Já para a base UNIFESP, os resultados não são tão positivos quanto os da base COPE, porém, considerando todos os aspectos já citados anteriormente em relação às 
dificuldades em se normalizar esta base e o número reduzido de imagens, gerando um hiperplano não tão assertivo, temos valores de acurácia consideráveis, mas que podem ser melhorados por meio de uma nova seleção de imagens ou utilizando-se diferentes métricas de avaliação dos hiperplanos gerados nos métodos de validação.

\subsection{Mapas $\mathbf{T}$ de dor}

Foi realizada a comparação entre as imagens artificiais geradas na navegação do hiperplano do MLDA através da construção dos Mapas T, descrita na seção anterior, para verificar quais regiões faciais se destacam nos momentos em que o recém-nascido está sofrendo experiências dolorosas.

Logo, por meio deste processo, foi utilizada a seguinte seleção de pares apresentada na Figura 12.

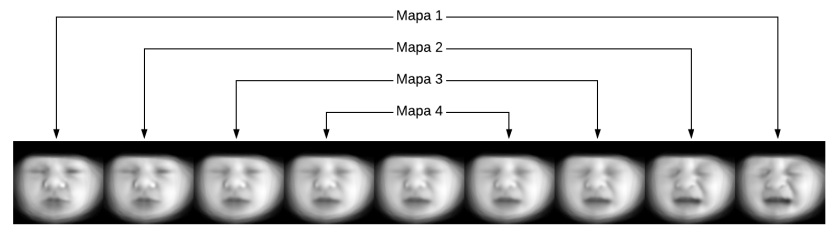

Figura 12. Seleção de pares para construção dos mapas.

Com as imagens devidamente selecionadas, foi gerado o mapa 1, que utilizou as imagens dos extremos da navegação do MLDA, para cada uma das bases. O mapa 1 foi escolhido dado que este compara as imagens dos extremos do hiperplano, ou seja, compara as imagens de face com mais dor e menos dor. O objetivo deste processo é identificar as regiões da face mais destacadas no momento de uma experiência dolorosa, primeiramente foi aplicado nos mapas um filtro da cor vermelha, mais precisamente uma multiplicação dos pixels da imagem original dos mapas por uma imagem de mesmo tamanho e cor sólida vermelha, sendo o resultado final da transformação, um mapa em que as regiões de maior destaque receberam tons de vermelho mais vivos, e as de menos destaque tons mais claros. Após isto, foi aplicada uma sobreposição dessas imagens, já transformadas em tons de vermelho, sobre as médias globais de ambas as bases.

Por meio deste processo, foram geradas as imagens das Figuras 13a e 13b para as bases COPE e UNIFESP, respectivamente.

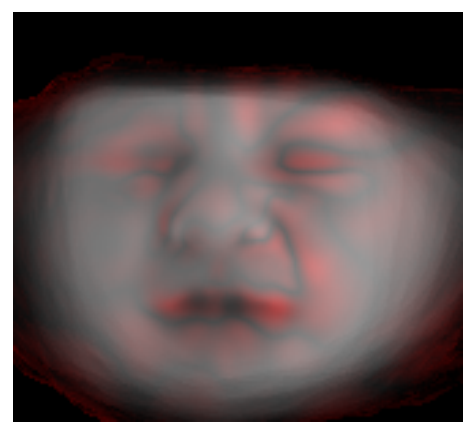

(a)

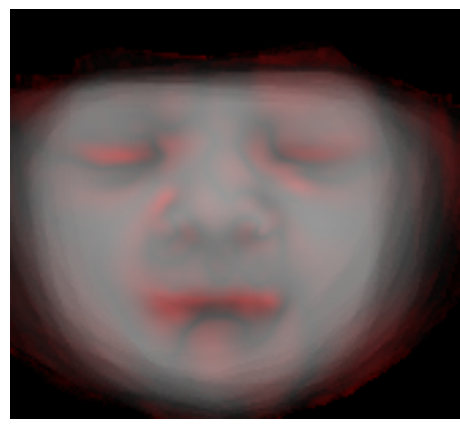

(b)

Figura 13. Regiões de destaque para a base COPE e UNIFESP. 
Analisando as Figuras 13a e 13b, vemos que o entorno da boca, quase toda a região orbitária, uma porção da região jugal (da bochecha), além da elevação das sobrancelhas e enrijecimento da glabela são partes da face que recebem destaque em detrimento das outras em momentos que um recém nascido está experienciando dor.

Desconsiderando as áreas externas à face, os pontos de destaque especificados anteriormente servem como informação estatística discriminante para profissionais da saúde, pois estes poderiam prestar uma maior atenção a estas regiões e provavelmente serem mais assertivos quanto a avaliação de dor em momentos de urgência. Estas imagens podem ser úteis também para a criação de algoritmos que fossem projetados de maneira a avaliar dor segundo essas regiões de destaque, ou seja, toda o processamento e análise recairia nestas regiões, ou então pesos maiores poderiam ser atribuídos a essas áreas. Há a possibilidade de se criar uma nova escala de dor, ou enriquecer as existentes, utilizando estas regiões como pontos de destaque.

\section{Conclusão}

Este trabalho descreveu um arcabouço computacional de reconhecimento de padrões faciais utilizando passos bem definidos de normalização e extração multivariada de informação. Para o processo de classificação, foram avaliados hiperplanos estatísticos gerados através da combinação PCA+MLDA, aplicada às bases de imagens faciais de recém-nascidos investigadas (COPE e UNIFESP), mostrando que podemos separar estas bases entre imagens de dor e não-dor utilizando estes métodos lineares. Os resultados de acurácia obtidos através do arcabouço construído neste trabalho atingiram níveis consideráveis, quando comparados com trabalhos da mesma área [Zhi et al. 2018, Zamzmi et al. 2018, Nanni et al. 2010], dado que para a base COPE a taxa de acurácia utilizando leave-one-out foi de mais de $95 \%$, ultrapassando os valores obtidos até então utilizando esta mesma base de imagens.

Outra contribuição foi a geração dos mapas T, pois estes descrevem quantitativamente as regiões mais discriminantes quando está se discutindo avaliação de dor em imagens faciais, como por exemplo, regiões próximas aos olhos e boca discriminam muito mais dor que o restante da face. Portanto, estes mapas podem auxiliar os profissionais a realizarem uma avaliação mais específica e assertiva sobre a atual situação do recémnascido.

Entretanto, deve-se destacar as seguintes limitações com relação ao arcabouço proposto. Foi utilizada uma seleção específica anterior à normalização e classificação, escolhendo apenas as imagens com ângulos próximos ao de perfil, sendo que pode-se realizar uma nova seleção, adicionando ou removendo imagens com diferentes angulações. $\mathrm{E}$ por fim, ambas as bases foram construídas utilizando bebês a termo, ou seja, ainda existe o desafio de avaliar imagens de recém-nascidos que se encontram em situações adversas, como por exemplo, bebês que necessitam de aparelhos extras no processo de recuperação dentro de uma UTI neonatal.

A partir deste trabalho, algumas possibilidades, que vão além do que foi apresentado aqui, podem ser executadas como trabalhos futuros, tais como: utilizar o arcabouço construído como backend de um sistema que monitora bebês recém-nascidos e notifica através de mensagens momentos de possíveis experiências de dor por parte dos indivíduos; Testar novos algoritmos de classificação utilizando o mesmo método de 
normalização, como, por exemplo, KNN, árvores de decisão, ou redes neurais.

\section{Referências}

Brahnam, S., Chuang, C.-F., Shih, F. Y., and Slack, M. R. (2006). Machine recognition and representation of neonatal facial displays of acute pain. Artificial intelligence in medicine, 36(3):211-222.

Fukunaga, K. (2013). Introduction to statistical pattern recognition. Elsevier.

Gibson, S. J. (2006). Eigenfit: A statistical learning approach to facial composites. PhD thesis, University of Kent.

Heiderich, T. M., Leslie, A. T. F. S., and Guinsburg (2015). Neonatal procedural pain can be assessed by computer software that has good sensitivity and specificity to detect facial movements. Acta Paediatrica, 104(2):e63-e69.

Johnson, R. A. and Wichern, D. W. (2006). Applied Multivariate Statistical Analysis. Prentice-Hall, Inc., Upper Saddle River, NJ, USA, 6 edition.

Luda, Nackley, A. G., Tchivileva, I. E., Shabalina, S. A., and Maixner, W. (2007). Genetic architecture of human pain perception. TRENDS in Genetics, 23(12):605-613.

Mansor, M. N. and Rejab, M. N. (2013). A computational model of the infant pain impressions with gaussian and nearest mean classifier. In Control System, Computing and Engineering (ICCSCE), 2013 IEEE International Conference on, pages 249-253. IEEE.

Nanni, L., Brahnam, S., and Lumini, A. (2010). A local approach based on a local binary patterns variant texture descriptor for classifying pain states. Expert Systems with Applications, 37(12):7888-7894.

Orona, P. A., Fabbro, D. A., Heiderich, T. M., Barros, M. C., Balda, R. C., Guinsburg, R., and Thomaz, C. E. (2019). Atlas of neonatal face images using triangular meshes. In Anais do XV Workshop de Visão Computacional, pages 19-24. SBC.

Taddio, A., Katz, J., Ilersich, A. L., and Koren, G. (1997). Effect of neonatal circumcision on pain response during subsequent routine vaccination. The lancet, 349(9052):599603.

Teruel, G. F., Heiderich, T. M., Guinsburg, R., and Thomaz, C. E. (2019). Análise e reconhecimento de dor em imagens $2 \mathrm{~d}$ frontais de recém-nascidos a termo e saudáveis. In Anais Estendidos do XIX Simpósio Brasileiro de Computação Aplicada à Saúde, pages $97-102$. SBC.

Thomaz, C. E., Kitani, E. C., and Gillies, D. F. (2006). A maximum uncertainty lda-based approach for limited sample size problems - with application to face recognition. Journal of the Brazilian Computer Society, 12(2):7-18.

Zamzmi, G., Goldgof, D., Kasturi, R., and Sun, Y. (2018). Neonatal pain expression recognition using transfer learning. arXiv preprint arXiv:1807.01631.

Zhi, R., Zamzmi, G., Goldgof, D., Ashmeade, T., and Sun, Y. (2018). Automatic infants' pain assessment by dynamic facial representation: Effects of profile view, gestational age, gender, and race. Journal of clinical medicine, 7(7):173. 